\title{
Coronavirus disease (COVID-19)
}

\section{Situation Report - 194}

Data as received by WHO from national authorities by 10:00 CEST, 1 August 2020

\section{Highlights}

The fourth meeting of the International Health Regulations (2005) Emergency Committee regarding COVID-19 was convened by the WHO Director-General on 31 July 2020. The Director-General declared that the outbreak of COVID-19 continues to constitute a public health emergency of international concern (PHEIC). The recommendations from the Emergency Committee highlight the need for response efforts to continue over the long term.

WHO has updated the interim guidance on Water, sanitation, hygiene (WASH), and waste management for SARS-CoV-2. The document provides additional details on risks associated with excreta and untreated sewage, on hand hygiene, on protecting WASH workers and on supporting the continuation and strengthening of WASH services, especially in underserved areas.

WHO has published a draft for Target Product Profiles (TPP) for COVID-19 therapeutics. The three sets of TPPs describe the preferred and minimally acceptable profiles for therapeutic agents for the treatment of those with COVID-19, ranging from mild through critically ill patients. Comments on this document are welcomed by submitting a comment form. All forms should be completed with the details of the individual or organization providing the comment.

For World Breastfeeding Week, WHO and UNICEF are calling on governments to protect and promote women's access to skilled breastfeeding counselling. This is a critical component of breastfeeding support and, amidst the COVID-19 pandemic, it is even more important to find innovative solutions to ensure that access to these essential services is not disrupted.

\section{Situation in numbers (by WHO Region)}

Total (new cases in last 24 hours)

\begin{tabular}{lrr}
\hline Globally & 17396943 cases (289 321) & 675060 deaths (6 142) \\
\hline Africa & 788448 cases (18 027) & 13545 deaths (311) \\
Americas & 9320330 cases (168 157) & 355217 deaths (4 096) \\
Eastern Mediterranean & 1544994 cases (11 637) & 40019 deaths (358) \\
Europe & 3357465 cases (22 550) & 212978 deaths (450) \\
South-East Asia & 2072194 cases (62 231) & 44900 deaths (869) \\
Western Pacific & 312771 cases (6 719) & 8388 deaths (58)
\end{tabular}




\section{Surveillance}

Figure 1. Number of confirmed* COVID-19 cases reported in the last seven days by country, territory or area, 26 July to 1 August **

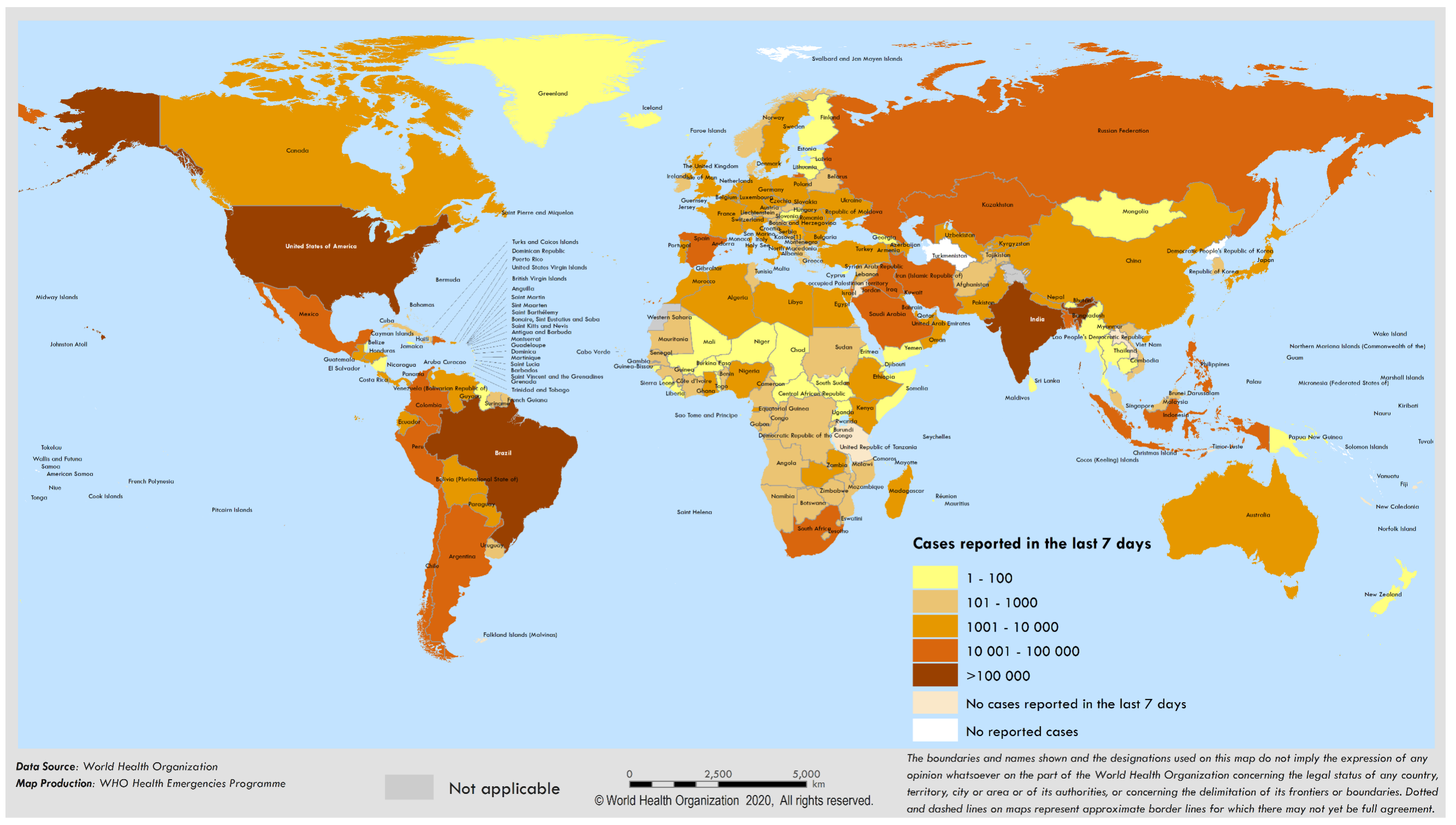

**See Annex 1 for data, table and figure notes. 
Figure 2. Number of confirmed COVID-19 cases, by date of report and WHO region, 30 December through 1 August**

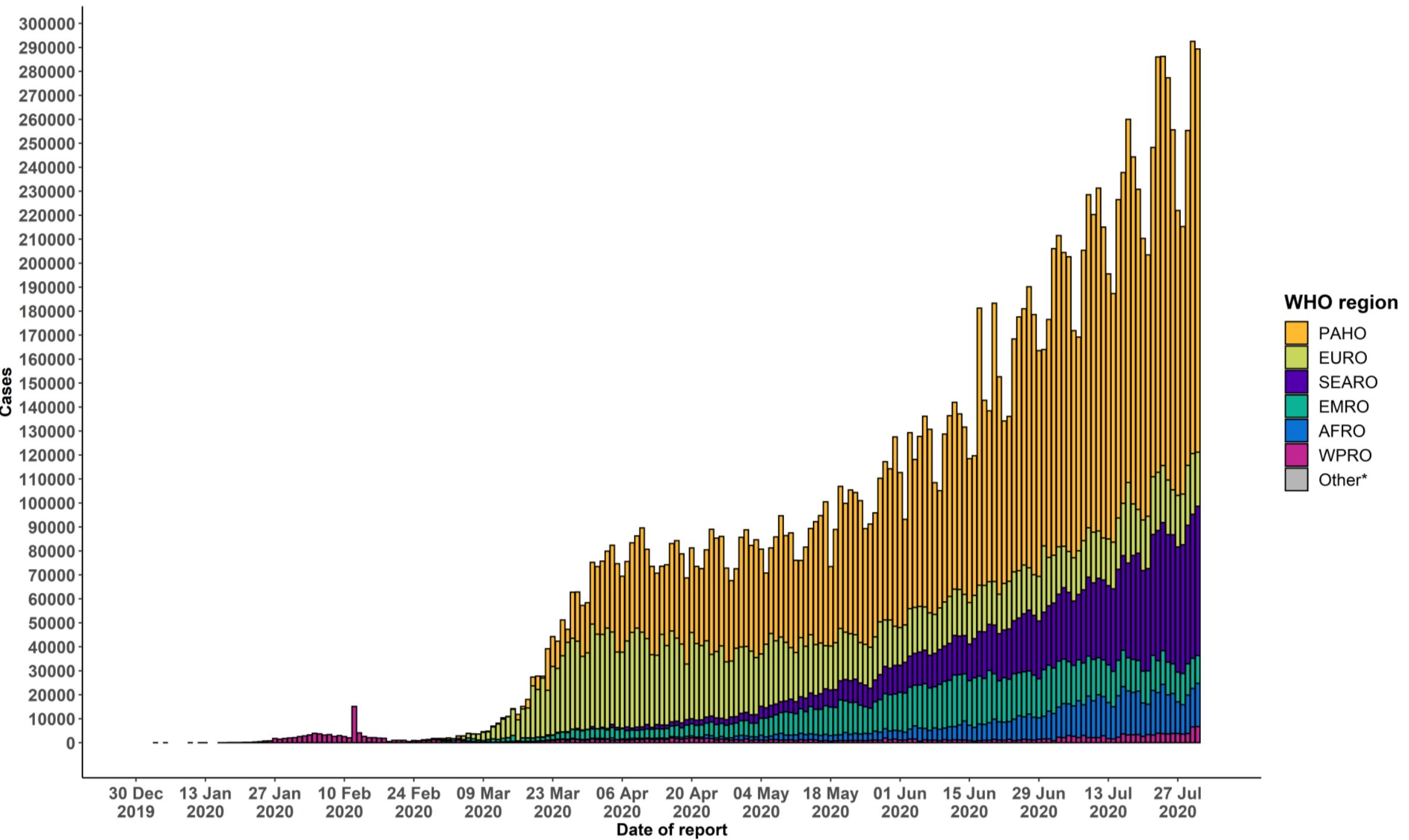

**See Annex 1 for data, table and figure notes. 
Table 1. Countries, territories or areas with reported laboratory-confirmed* COVID-19 cases and deaths, by WHO region. Data as of 10 AM CEST, 1 August $2020^{* *}$

\begin{tabular}{|c|c|c|c|c|c|c|}
\hline Reporting Country/Territory/Area & $\begin{array}{l}\text { Total confirmed } \\
\text { cases }\end{array}$ & $\begin{array}{l}\text { Total confirmed } \\
\text { new cases }\end{array}$ & Total deaths & Total new deaths & $\begin{array}{l}\text { Transmission } \\
\text { classification }\end{array}$ & $\begin{array}{l}\text { Days since last } \\
\text { reported case }\end{array}$ \\
\hline \multicolumn{7}{|l|}{ Africa } \\
\hline South Africa & 493183 & 11014 & 8005 & 193 & Community transmission & 0 \\
\hline Nigeria & 43151 & 462 & 879 & 1 & Community transmission & 0 \\
\hline Ghana & 35501 & 359 & 182 & 7 & Community transmission & 0 \\
\hline Algeria & 30394 & 563 & 1210 & 10 & Community transmission & 0 \\
\hline Kenya & 20636 & 723 & 341 & 16 & Community transmission & 0 \\
\hline Ethiopia & 17530 & 915 & 274 & 11 & Community transmission & 0 \\
\hline Cameroon & 17255 & 0 & 391 & 0 & Community transmission & 2 \\
\hline Côte d'Ivoire & 16047 & 69 & 102 & 2 & Community transmission & 0 \\
\hline Madagascar & 10868 & 120 & 106 & 1 & Community transmission & 0 \\
\hline Senegal & 10232 & 126 & 205 & 1 & Community transmission & 0 \\
\hline Democratic Republic of the Congo & 9069 & 60 & 214 & 0 & Community transmission & 0 \\
\hline Gabon & 7352 & 0 & 49 & 0 & Community transmission & 2 \\
\hline Guinea & 7308 & 66 & 46 & 0 & Community transmission & 0 \\
\hline Mauritania & 6310 & 40 & 157 & 1 & Community transmission & 0 \\
\hline Zambia & 5963 & 408 & 151 & 2 & Community transmission & 0 \\
\hline Equatorial Guinea & 4821 & 2471 & 83 & 42 & Community transmission & 0 \\
\hline Central African Republic & 4608 & 3 & 59 & 0 & Community transmission & 0 \\
\hline Malawi & 4078 & 97 & 114 & 5 & Community transmission & 0 \\
\hline Congo & 3376 & 0 & 56 & 0 & Community transmission & 1 \\
\hline Zimbabwe & 3169 & 77 & 67 & 14 & Clusters of cases & 0 \\
\hline Eswatini & 2648 & 71 & 40 & 0 & Community transmission & 0 \\
\hline
\end{tabular}




\begin{tabular}{|c|c|c|c|c|c|c|}
\hline Reporting Country/Territory/Area & $\begin{array}{l}\text { Total confirmed } \\
\text { cases }\end{array}$ & $\begin{array}{l}\text { Total confirmed } \\
\text { new cases }\end{array}$ & Total deaths & Total new deaths & $\begin{array}{l}\text { Transmission } \\
\text { classification }\end{array}$ & $\begin{array}{l}\text { Days since last } \\
\text { reported case }\end{array}$ \\
\hline Mali & 2522 & 0 & 124 & 0 & Community transmission & 1 \\
\hline Cabo Verde & 2451 & 33 & 23 & 0 & Clusters of cases & 0 \\
\hline South Sudan & 2352 & 30 & 46 & 0 & Community transmission & 0 \\
\hline Namibia & 2129 & 77 & 10 & 0 & Clusters of cases & 0 \\
\hline Rwanda & 2022 & 28 & 5 & 0 & Clusters of cases & 0 \\
\hline Guinea-Bissau & 1981 & 0 & 27 & 1 & Community transmission & 2 \\
\hline Mozambique & 1864 & 56 & 11 & 0 & Community transmission & 0 \\
\hline Sierra Leone & 1823 & 5 & 67 & 0 & Community transmission & 0 \\
\hline Benin & 1805 & 0 & 36 & 0 & Community transmission & 3 \\
\hline Liberia & 1186 & 5 & 75 & 2 & Community transmission & 0 \\
\hline Uganda & 1154 & 7 & 3 & 1 & Clusters of cases & 0 \\
\hline Burkina Faso & 1138 & 21 & 53 & 0 & Community transmission & 0 \\
\hline Niger & 1136 & 2 & 69 & 0 & Community transmission & 0 \\
\hline Angola & 1109 & 0 & 51 & 0 & Clusters of cases & 1 \\
\hline Chad & 936 & 1 & 75 & 0 & Community transmission & 0 \\
\hline Togo & 927 & 19 & 18 & 0 & Community transmission & 0 \\
\hline Sao Tome and Principe & 871 & 1 & 15 & 0 & Clusters of cases & 0 \\
\hline Botswana & 804 & 0 & 2 & 0 & Clusters of cases & 2 \\
\hline Lesotho & 604 & 0 & 13 & 0 & Clusters of cases & 1 \\
\hline United Republic of Tanzania & 509 & 0 & 21 & 0 & Community transmission & 85 \\
\hline Gambia & 498 & 95 & 9 & 1 & Sporadic cases & 0 \\
\hline Burundi & 387 & 0 & 1 & 0 & Clusters of cases & 1 \\
\hline Comoros & 382 & 0 & 7 & 0 & Community transmission & 1 \\
\hline
\end{tabular}




\begin{tabular}{|c|c|c|c|c|c|c|}
\hline Reporting Country/Territory/Area & $\begin{array}{l}\text { Total confirmed } \\
\text { cases }\end{array}$ & $\begin{array}{l}\text { Total confirmed } \\
\text { new cases }\end{array}$ & Total deaths & Total new deaths & $\begin{array}{l}\text { Transmission } \\
\text { classification }\end{array}$ & $\begin{array}{l}\text { Days since last } \\
\text { reported case }\end{array}$ \\
\hline Mauritius & 344 & 0 & 10 & 0 & Sporadic cases & 7 \\
\hline Eritrea & 279 & 0 & 0 & 0 & Sporadic cases & 1 \\
\hline Seychelles & 114 & 0 & 0 & 0 & Sporadic cases & 4 \\
\hline \multicolumn{7}{|l|}{ Territories ${ }^{\mathrm{ii}}$} \\
\hline Mayotte & 2962 & 0 & 39 & 0 & Clusters of cases & 1 \\
\hline Réunion & 660 & 3 & 4 & 0 & Clusters of cases & 0 \\
\hline \multicolumn{7}{|l|}{ Americas } \\
\hline United States of America & 4456389 & 67823 & 151265 & 1211 & Community transmission & 0 \\
\hline Brazil & 2610102 & 57837 & 91263 & 1129 & Community transmission & 0 \\
\hline Mexico & 416179 & 7730 & 46000 & 639 & Community transmission & 0 \\
\hline Peru & 407492 & 6809 & 19021 & 205 & Community transmission & 0 \\
\hline Chile & 355667 & 2131 & 9457 & 80 & Community transmission & 0 \\
\hline Colombia & 286020 & 9965 & 9810 & 356 & Community transmission & 0 \\
\hline Argentina & 185373 & 6377 & 3466 & 155 & Community transmission & 0 \\
\hline Canada & 115799 & 329 & 8929 & 12 & Community transmission & 0 \\
\hline Ecuador & 85355 & 985 & 5702 & 45 & Community transmission & 0 \\
\hline Bolivia (Plurinational State of) & 75234 & 1700 & 2894 & 86 & Community transmission & 0 \\
\hline Dominican Republic & 69649 & 1734 & 1160 & 14 & Community transmission & 0 \\
\hline Panama & 64191 & 922 & 1397 & 23 & Community transmission & 0 \\
\hline Guatemala & 49789 & 963 & 1924 & 57 & Community transmission & 0 \\
\hline Honduras & 41426 & 482 & 1312 & 53 & Community transmission & 0 \\
\hline Venezuela (Bolivarian Republic of) & 17859 & 700 & 158 & 4 & Community transmission & 0 \\
\hline Costa Rica & 17290 & 490 & 140 & 7 & Community transmission & 0 \\
\hline El Salvador & 16632 & 402 & 448 & 9 & Community transmission & 0 \\
\hline
\end{tabular}




\begin{tabular}{|c|c|c|c|c|c|c|}
\hline Reporting Country/Territory/Area & $\begin{array}{l}\text { Total confirmed } \\
\text { cases }\end{array}$ & $\begin{array}{l}\text { Total confirmed } \\
\text { new cases }\end{array}$ & Total deaths & Total new deaths & $\begin{array}{l}\text { Transmission } \\
\text { classification }^{\mathrm{i}}\end{array}$ & $\begin{array}{l}\text { Days since last } \\
\text { reported case }\end{array}$ \\
\hline Haiti & 7412 & 34 & 161 & 2 & Community transmission & 0 \\
\hline Paraguay & 5207 & 341 & 47 & 1 & Community transmission & 0 \\
\hline Nicaragua & 3080 & 0 & 116 & 0 & Community transmission & 3 \\
\hline Cuba & 2608 & 11 & 87 & 0 & Clusters of cases & 0 \\
\hline Suriname & 1650 & 43 & 26 & 0 & Clusters of cases & 0 \\
\hline Uruguay & 1243 & 6 & 35 & 0 & Clusters of cases & 0 \\
\hline Jamaica & 864 & 8 & 10 & 0 & Clusters of cases & 0 \\
\hline Bahamas & 508 & 24 & 14 & 3 & Clusters of cases & 0 \\
\hline Guyana & 401 & 3 & 20 & 0 & Clusters of cases & 0 \\
\hline Trinidad and Tobago & 169 & 12 & 8 & 0 & Sporadic cases & 0 \\
\hline Barbados & 110 & 0 & 7 & 0 & Clusters of cases & 4 \\
\hline Antigua and Barbuda & 91 & 0 & 3 & 0 & Clusters of cases & 1 \\
\hline Saint Vincent and the Grenadines & 54 & 2 & 0 & 0 & Sporadic cases & 0 \\
\hline Belize & 48 & 0 & 2 & 0 & Sporadic cases & 6 \\
\hline Saint Lucia & 25 & 0 & 0 & 0 & Sporadic cases & 1 \\
\hline Grenada & 24 & 0 & 0 & 0 & Clusters of cases & 1 \\
\hline Dominica & 18 & 0 & 0 & 0 & Clusters of cases & 49 \\
\hline Saint Kitts and Nevis & 17 & 0 & 0 & 0 & No cases & 21 \\
\hline \multicolumn{7}{|l|}{ Territories ${ }^{\mathrm{ii}}$} \\
\hline Puerto Rico & 16781 & 209 & 219 & 5 & Community transmission & 0 \\
\hline French Guiana & 7799 & 71 & 43 & 0 & Community transmission & 0 \\
\hline United States Virgin Islands & 406 & 8 & 8 & 0 & Community transmission & 0 \\
\hline Martinique & 269 & 0 & 15 & 0 & Clusters of cases & 7 \\
\hline Guadeloupe & 244 & 0 & 14 & 0 & Clusters of cases & 2 \\
\hline
\end{tabular}




\begin{tabular}{|c|c|c|c|c|c|c|}
\hline Reporting Country/Territory/Area & $\begin{array}{l}\text { Total confirmed } \\
\text { cases }\end{array}$ & $\begin{array}{l}\text { Total confirmed } \\
\text { new cases }\end{array}$ & Total deaths & Total new deaths & $\begin{array}{l}\text { Transmission } \\
\text { classification }^{\mathrm{i}}\end{array}$ & $\begin{array}{l}\text { Days since last } \\
\text { reported case }\end{array}$ \\
\hline Cayman Islands & 203 & 0 & 1 & 0 & Sporadic cases & 17 \\
\hline Bermuda & 156 & 0 & 9 & 0 & Sporadic cases & 2 \\
\hline Sint Maarten & 128 & 2 & 15 & 0 & Community transmission & 0 \\
\hline Aruba & 120 & 1 & 3 & 0 & Sporadic cases & 0 \\
\hline Turks and Caicos Islands & 107 & 3 & 2 & 0 & Clusters of cases & 0 \\
\hline Saint Martin & 53 & 0 & 3 & 0 & Sporadic cases & 2 \\
\hline Curaçao & 29 & 0 & 1 & 0 & Sporadic cases & 7 \\
\hline Falkland Islands (Malvinas) & 13 & 0 & 0 & 0 & No cases & 97 \\
\hline Montserrat & 13 & 0 & 1 & 0 & No cases & 5 \\
\hline Bonaire, Sint Eustatius and Saba & 11 & 0 & 0 & 0 & Sporadic cases & 8 \\
\hline British Virgin Islands & 8 & 0 & 1 & 0 & No cases & 77 \\
\hline Saint Barthélemy & 8 & 0 & 0 & 0 & No cases & 2 \\
\hline Saint Pierre and Miquelon & 4 & 0 & 0 & 0 & Sporadic cases & 15 \\
\hline Anguilla & 3 & 0 & 0 & 0 & No cases & 119 \\
\hline \multicolumn{7}{|l|}{ Eastern Mediterranean } \\
\hline Iran (Islamic Republic of) & 304204 & 2674 & 16766 & 197 & Community transmission & 0 \\
\hline Pakistan & 278305 & 0 & 5951 & 0 & Clusters of cases & 1 \\
\hline Saudi Arabia & 275905 & 1686 & 2866 & -3 & Clusters of cases & 0 \\
\hline Iraq & 124609 & 3346 & 4741 & 70 & Community transmission & 0 \\
\hline Qatar & 110695 & 235 & 174 & 3 & Community transmission & 0 \\
\hline Egypt & 94078 & 321 & 4805 & 31 & Clusters of cases & 0 \\
\hline Oman & 79159 & 0 & 421 & 0 & Community transmission & 1 \\
\hline Kuwait & 66957 & 428 & 447 & 2 & Clusters of cases & 0 \\
\hline United Arab Emirates & 60506 & 283 & 351 & 2 & Community transmission & 0 \\
\hline
\end{tabular}




\begin{tabular}{|c|c|c|c|c|c|c|}
\hline Reporting Country/Territory/Area & $\begin{array}{l}\text { Total confirmed } \\
\text { cases }\end{array}$ & $\begin{array}{l}\text { Total confirmed } \\
\text { new cases }\end{array}$ & Total deaths & Total new deaths & $\begin{array}{l}\text { Transmission } \\
\text { classification }\end{array}$ & $\begin{array}{l}\text { Days since last } \\
\text { reported case }\end{array}$ \\
\hline Bahrain & 40982 & 227 & 148 & 2 & Clusters of cases & 0 \\
\hline Afghanistan & 36710 & 168 & 1283 & 12 & Clusters of cases & 0 \\
\hline Morocco & 24322 & 1063 & 353 & 7 & Clusters of cases & 0 \\
\hline Sudan & 11644 & 148 & 746 & 21 & Community transmission & 0 \\
\hline Djibouti & 5081 & 0 & 58 & 0 & Clusters of cases & 2 \\
\hline Lebanon & 4555 & 221 & 59 & 2 & Clusters of cases & 0 \\
\hline Libya & 3621 & 399 & 74 & 3 & Clusters of cases & 0 \\
\hline Somalia & 3212 & 0 & 93 & 0 & Sporadic cases & 3 \\
\hline Yemen & 1732 & 2 & 494 & 6 & Community transmission & 0 \\
\hline Tunisia & 1535 & 21 & 50 & 0 & Sporadic cases & 0 \\
\hline Jordan & 1193 & 2 & 11 & 0 & Clusters of cases & 0 \\
\hline Syrian Arab Republic & 757 & 19 & 43 & 2 & Community transmission & 0 \\
\hline \multicolumn{7}{|l|}{ Territories ${ }^{\mathrm{ii}}$} \\
\hline occupied Palestinian territory & 15232 & 394 & 85 & 1 & Clusters of cases & 0 \\
\hline \multicolumn{7}{|l|}{ Europe } \\
\hline Russian Federation & 845443 & 5462 & 14058 & 95 & Clusters of cases & 0 \\
\hline The United Kingdom & 303185 & 880 & 46119 & 120 & Community transmission & 0 \\
\hline Spain & 288522 & 3092 & 28445 & 2 & Clusters of cases & 0 \\
\hline Italy & 247537 & 379 & 35141 & 9 & Clusters of cases & 0 \\
\hline Turkey & 230873 & 982 & 5691 & 17 & Community transmission & 0 \\
\hline Germany & 209653 & 955 & 9148 & 7 & Clusters of cases & 0 \\
\hline France & 175920 & 1272 & 30147 & 11 & Clusters of cases & 0 \\
\hline Kazakhstan & 90367 & 1289 & 793 & 0 & Clusters of cases & 0 \\
\hline Sweden & 80422 & 322 & 5743 & 4 & Community transmission & 0 \\
\hline
\end{tabular}




\begin{tabular}{|c|c|c|c|c|c|c|}
\hline Reporting Country/Territory/Area & $\begin{array}{l}\text { Total confirmed } \\
\text { cases }\end{array}$ & $\begin{array}{l}\text { Total confirmed } \\
\text { new cases }\end{array}$ & Total deaths & Total new deaths & $\begin{array}{l}\text { Transmission } \\
\text { classification }\end{array}$ & $\begin{array}{l}\text { Days since last } \\
\text { reported case }\end{array}$ \\
\hline Ukraine & 71056 & 1172 & 1709 & 16 & Community transmission & 0 \\
\hline Belgium & 68658 & 0 & 9841 & 0 & Community transmission & 1 \\
\hline Israel & 68492 & 0 & 493 & 0 & Community transmission & 1 \\
\hline Belarus & 67808 & 0 & 559 & 0 & Community transmission & 1 \\
\hline Netherlands & 54301 & 338 & 6147 & 0 & Community transmission & 0 \\
\hline Portugal & 51072 & 204 & 1735 & 8 & Clusters of cases & 0 \\
\hline Romania & 50886 & 1295 & 2343 & 39 & Community transmission & 0 \\
\hline Poland & 45688 & 657 & 1716 & 7 & Community transmission & 0 \\
\hline Armenia & 38841 & 291 & 749 & 11 & Community transmission & 0 \\
\hline Kyrgyzstan & 36299 & 494 & 1397 & 19 & Clusters of cases & 0 \\
\hline Switzerland & 34933 & 0 & 1703 & 0 & Community transmission & 1 \\
\hline Azerbaijan & 31878 & 318 & 448 & 7 & Clusters of cases & 0 \\
\hline Ireland & 26065 & 38 & 1763 & 0 & Clusters of cases & 0 \\
\hline Serbia & 25552 & 339 & 573 & 8 & Community transmission & 0 \\
\hline Republic of Moldova & 24733 & 390 & 778 & 7 & Community transmission & 0 \\
\hline Uzbekistan & 24304 & 746 & 143 & 6 & Clusters of cases & 0 \\
\hline Austria & 21098 & 89 & 718 & 0 & Community transmission & 0 \\
\hline Czechia & 16574 & 232 & 382 & 3 & Clusters of cases & 0 \\
\hline Denmark & 13789 & 64 & 615 & 0 & Community transmission & 0 \\
\hline Bulgaria & 11690 & 270 & 383 & 9 & Clusters of cases & 0 \\
\hline Bosnia and Herzegovina & 11442 & 0 & 324 & 0 & Community transmission & 1 \\
\hline North Macedonia & 10762 & 145 & 486 & 6 & Clusters of cases & 0 \\
\hline Norway & 9208 & 36 & 255 & 0 & Clusters of cases & 0 \\
\hline Finland & 7432 & 9 & 329 & 0 & Clusters of cases & 0 \\
\hline
\end{tabular}




\begin{tabular}{|c|c|c|c|c|c|c|}
\hline Reporting Country/Territory/Area & $\begin{array}{l}\text { Total confirmed } \\
\text { cases }\end{array}$ & $\begin{array}{l}\text { Total confirmed* } \\
\text { new cases }\end{array}$ & Total deaths & Total new deaths & $\begin{array}{l}\text { Transmission } \\
\text { classification }\end{array}$ & $\begin{array}{l}\text { Days since last } \\
\text { reported case }\end{array}$ \\
\hline Tajikistan & 7409 & 43 & 60 & 0 & Pending & 0 \\
\hline Luxembourg & 6695 & 79 & 114 & 0 & Community transmission & 0 \\
\hline Albania & 5276 & 79 & 157 & 3 & Clusters of cases & 0 \\
\hline Croatia & 5138 & 67 & 145 & 1 & Clusters of cases & 0 \\
\hline Hungary & 4505 & 0 & 596 & 0 & Community transmission & 1 \\
\hline Greece & 4477 & 76 & 206 & 3 & Clusters of cases & 0 \\
\hline Montenegro & 3184 & 73 & 49 & 1 & Clusters of cases & 0 \\
\hline Slovakia & 2292 & 27 & 29 & 1 & Clusters of cases & 0 \\
\hline Slovenia & 2165 & 26 & 117 & 0 & Clusters of cases & 0 \\
\hline Lithuania & 2075 & 13 & 80 & 0 & Community transmission & 0 \\
\hline Estonia & 2064 & 13 & 69 & 0 & Sporadic cases & 0 \\
\hline Iceland & 1885 & 13 & 10 & 0 & Community transmission & 0 \\
\hline Latvia & 1231 & 3 & 32 & 1 & Clusters of cases & 0 \\
\hline Georgia & 1171 & 3 & 17 & 0 & Sporadic cases & 0 \\
\hline Cyprus & 1114 & 30 & 19 & 0 & Clusters of cases & 0 \\
\hline Andorra & 925 & 3 & 66 & 14 & Community transmission & 0 \\
\hline Malta & 824 & 10 & 9 & 0 & Sporadic cases & 0 \\
\hline San Marino & 716 & 0 & 42 & 0 & Community transmission & 22 \\
\hline Monaco & 99 & 0 & 1 & 0 & Sporadic cases & 55 \\
\hline Liechtenstein & 89 & 0 & 1 & 0 & Sporadic cases & 1 \\
\hline Holy See & 12 & 0 & 0 & 0 & Sporadic cases & 86 \\
\hline \multicolumn{7}{|l|}{ Territoriesii } \\
\hline Kosovo $^{[1]}$ & 8287 & 226 & 217 & 15 & Community transmission & 0 \\
\hline
\end{tabular}




\begin{tabular}{|c|c|c|c|c|c|c|}
\hline Reporting Country/Territory/Area & $\begin{array}{l}\text { Total confirmed } \\
\text { cases }\end{array}$ & $\begin{array}{l}\text { Total confirmed } \\
\text { new cases }\end{array}$ & Total deaths & Total new deaths & $\begin{array}{l}\text { Transmission } \\
\text { classification }\end{array}$ & $\begin{array}{l}\text { Days since last } \\
\text { reported case }\end{array}$ \\
\hline Isle of Man & 336 & 0 & 24 & 0 & No cases & 71 \\
\hline Jersey & 335 & 0 & 31 & 0 & Community transmission & 2 \\
\hline Guernsey & 252 & 0 & 13 & 0 & Community transmission & 90 \\
\hline Faroe Islands & 225 & 5 & 0 & 0 & Sporadic cases & 0 \\
\hline Gibraltar & 187 & 1 & 0 & 0 & Clusters of cases & 0 \\
\hline Greenland & 14 & 0 & 0 & 0 & No cases & 3 \\
\hline \multicolumn{7}{|l|}{ South-East Asia } \\
\hline India & 1695988 & 57118 & 36511 & 764 & Clusters of cases & 0 \\
\hline Bangladesh & 237661 & 2772 & 3111 & 28 & Community transmission & 0 \\
\hline Indonesia & 108376 & 2040 & 5131 & 73 & Community transmission & 0 \\
\hline Nepal & 19771 & 224 & 56 & 4 & Clusters of cases & 0 \\
\hline Maldives & 3793 & 74 & 16 & 0 & Clusters of cases & 0 \\
\hline Thailand & 3312 & 2 & 58 & 0 & Clusters of cases & 0 \\
\hline Sri Lanka & 2815 & 1 & 11 & 0 & Clusters of cases & 0 \\
\hline Myanmar & 353 & 0 & 6 & 0 & Clusters of cases & 2 \\
\hline Bhutan & 101 & 0 & 0 & 0 & Sporadic cases & 2 \\
\hline Timor-Leste & 24 & 0 & 0 & 0 & No cases & 99 \\
\hline \multicolumn{7}{|l|}{ Western Pacific } \\
\hline Philippines & 93354 & 3980 & 2023 & 40 & Community transmission & 0 \\
\hline China & 88122 & 166 & 4668 & 2 & Clusters of cases & 0 \\
\hline Singapore & 52205 & 396 & 27 & 0 & Clusters of cases & 0 \\
\hline Japan & 35836 & 1464 & 1011 & 5 & Clusters of cases & 0 \\
\hline Australia & 16905 & 602 & 196 & 7 & Clusters of cases & 0 \\
\hline
\end{tabular}




\begin{tabular}{|c|c|c|c|c|c|c|}
\hline Reporting Country/Territory/Area & $\begin{array}{l}\text { Total confirmed } \\
\text { cases }\end{array}$ & $\begin{array}{l}\text { Total confirmed } \\
\text { new cases }\end{array}$ & Total deaths & Total new deaths & $\begin{array}{l}\text { Transmission } \\
\text { classification }^{\mathrm{i}}\end{array}$ & $\begin{array}{l}\text { Days since last } \\
\text { reported case }\end{array}$ \\
\hline Republic of Korea & 14336 & 31 & 301 & 0 & Clusters of cases & 0 \\
\hline Malaysia & 8976 & 12 & 125 & 1 & Clusters of cases & 0 \\
\hline New Zealand & 1212 & 2 & 22 & 0 & Clusters of cases & 0 \\
\hline Viet Nam & 558 & 49 & 2 & 2 & Clusters of cases & 0 \\
\hline Mongolia & 291 & 0 & 0 & 0 & Sporadic cases & 2 \\
\hline Cambodia & 239 & 5 & 0 & 0 & Sporadic cases & 0 \\
\hline Brunei Darussalam & 141 & 0 & 3 & 0 & No cases & 85 \\
\hline Papua New Guinea & 72 & 9 & 2 & 0 & Sporadic cases & 0 \\
\hline Fiji & 27 & 0 & 1 & 1 & Sporadic cases & 11 \\
\hline Lao People's Democratic Republic & 20 & 0 & 0 & 0 & Sporadic cases & 7 \\
\hline \multicolumn{7}{|l|}{ Territories ${ }^{\mathrm{ii}}$} \\
\hline Guam & 351 & 3 & 5 & 0 & Clusters of cases & 0 \\
\hline French Polynesia & 62 & 0 & 0 & 0 & Sporadic cases & 34 \\
\hline $\begin{array}{l}\text { Northern Mariana Islands } \\
\text { (Commonwealth of the) }\end{array}$ & 42 & 0 & 2 & 0 & Pending & 1 \\
\hline New Caledonia & 22 & 0 & 0 & 0 & Sporadic cases & 17 \\
\hline Subtotal for all regions & 17396202 & 289321 & 675047 & 6142 & & \\
\hline Other $^{\dagger}$ & 741 & 0 & 13 & 0 & Not applicable & - \\
\hline Grand total & 17396943 & 289321 & 675060 & 6142 & & \\
\hline
\end{tabular}

**See Annex 1 for data, table and figure notes. 


\section{Technical guidance and other resources}

- To view all technical guidance documents regarding COVID-19, please go to this webpage.

- Updates from WHO regional offices

- WHO AFRO

- WHO EMRO

- WHO EURO
- WHOPAHO

- WHOSEARO

- WHOWPRO

- Research and Development

- Online courses on COVID-19 and in additional national languages

- The Strategic Preparedness and Response Plan(SPRP) outlining the support the international community can provide to all countries to prepare and respond to the virus

- WHO Coronavirus Disease (COVID-19) Dashboard

- Weekly COVID-19 Operations Updates

\section{Recommendations and advice for the public}

- Protect yourself

- Questions and answers

- Travel advice

- EPI-WIN: tailored information for individuals, organizations and communities

\section{Case definitions}

WHO periodically updates the Global Surveillance for human infection with coronavirus disease (COVID-19) document which includes surveillance definitions.

\section{Definition of COVID-19 death}

A COVID-19 death is defined for surveillance purposes as a death resulting from a clinically compatible illness in a probable or confirmed COVID-19 case, unless there is a clear alternative cause of death that cannot be related to COVID-19 disease (e.g. trauma). There should be no period of complete recovery between the illness and death.

Further guidance for certification and classification (coding) of COVID-19 as cause of death is available here and here. 


\section{Annex 1: Data, table and figure notes}

Caution must be taken when interpreting all data presented. Differences are to be expected between information products published by WHO, national public health authorities, and other sources using different inclusion criteria and different data cut-off times. While steps are taken to ensure accuracy and reliability, all data are subject to continuous verification and change. Case detection, definitions, testing strategies, reporting practice, and lag times differ between countries/territories/areas. These factors, amongst others, influence the counts presented, with variable underestimation of true case and death counts, and variable delays to reflecting these data at global level.

The designations employed, and the presentation of these materials do not imply the expression of any opinion whatsoever on the part of WHO concerning the legal status of any country, territory or area or of its authorities, or concerning the delimitation of its frontiers or boundaries. Dotted and dashed lines on maps represent approximate border lines for which there may not yet be full agreement. Countries, territories and areas are arranged under the administering WHO region.

The mention of specific companies or of certain manufacturers' products does not imply that they are endorsed or recommended by WHO in preference to others of a similar nature that are not mentioned. Errors and omissions excepted, the names of proprietary products are distinguished by initial capital letters.

${ }^{[1]}$ All references to Kosovo should be understood to be in the context of the United Nations Security Council resolution 1244 (1999). In the map, number of cases of Serbia and Kosovo (UNSCR 1244, 1999) have been aggregated for visualization purposes.

*A small number of countries/territories/areas report combined probable and laboratory-confirmed cases; efforts are underway to identify these for notation in the data table. ${ }^{\dagger}$ Other: includes cases reported from international conveyances.

Due to the recent trend of countries conducting data reconciliation exercises which remove large numbers of cases or deaths from their total counts, WHO will now display such data as negative numbers in the "new cases" / "new deaths" columns as appropriate. This will aid readers in identifying when such adjustments occur. When additional details become available that allow the subtractions to be suitably apportioned to previous days, graphics will be updated accordingly. Prior situation reports will not be edited; see covid19. who.int for the most up-to-date data.

\section{Additional table notes}

i Transmission classification is based on a process of country/territory/area self-reporting. Classifications are reviewed on a weekly basis and may be revised as new information becomes available. Differing degrees of transmission may be present within countries/territories/areas; classification is based on the highest category reported within a country/territory/area. Categories:

- No cases: with no confirmed cases;

- Sporadic cases: with one or more cases, imported or locally detected;

- Clusters of cases: experiencing cases, clustered in time, geographic location and/or by common exposures; 
- Community transmission: experiencing larger outbreaks of local transmission defined through an assessment of factors including, but not limited to: large numbers of cases not linkable to transmission chains; large numbers of cases from sentinel lab surveillance; and/or multiple unrelated clusters in several areas of the country/territory/area;

- Pending: transmission classification has not been reported to WHO.

ii "Territories" include territories, areas, overseas dependencies and other jurisdictions of similar status.

\section{Country, territory, or area-specific notes, updates and errata}

- Update 1 August 2020, Austria: Counts adjusted retrospectively by national authorities: case and death counts adjusted from 26 February to 22 March and from 22 July to 24 July. First case and first death also adjusted.

- Update 1 August 2020, Belgium: Counts adjusted retrospectively by national authorities: case and death counts from 2 July to 31 July.

- Update 1 August 2020, Bulgaria: Counts adjusted retrospectively by national authorities: case and death counts adjusted from 8 March to 21 March and 11 June.

- Update 1 August 2020, Croatia: Counts adjusted retrospectively by national authorities: case and death counts adjusted from 26 February to 22 March and 30 July.

- Update 1 August 2020, Germany: Counts adjusted retrospectively by national authorities: case and death counts adjusted from 28 February to 22 March, on 1 May, 14 July and 30-31 July. First death also adjusted.

- Update 1 August 2020, Greece: Counts adjusted retrospectively by national authorities: case and death counts adjusted from 27 February to 22 March and from 3 June to 8 June. First case also adjusted.

- Update 1 August 2020, Hungary: Counts adjusted retrospectively by national authorities: case and death counts adjusted from 5 March to 22 March and on 8 July. First case also adjusted.

- Update 1 August 2020, Netherlands: Counts adjusted retrospectively by national authorities: case and death counts adjusted from 27 February to 22 March and on 16 June. First case also adjusted.

- Update 1 August 2020, Poland: Counts adjusted retrospectively by national authorities: case and death counts adjusted from 4 March to 22 March and on 4 June. First case and first death also adjusted. 